\title{
考虑弹塑性阶段的结合面法向接触阻尼分形模型*
}

\author{
陈永会 张学良 温淑花 兰国生 \\ (太原科技大学机械工程学院 太原 030024)
}

\begin{abstract}
摘要: 结合面法向接触阻尼建模时, 只考虑微凸体弹性变形时的弹性能和塑性变形时的损耗能是不完全的, 因为微凸体存在 弹塑性变形情况。将微凸体弹塑性变形时的法向接触载荷 “分离” 为弹性载荷和塑性载荷两部分, 得到微凸体弹塑性变形时 的弹性能和损耗能, 建立更加完善、合理的结合面法向接触阻尼模型。将结合面法向接触动力学模型等效为弹簧和黏性阻尼 器, 建立包括微凸体三种变形状况的结合面法向接触阻尼损耗因子和法向接触阻尼系数的解析模型, 并量纲一化处理。仿真 结果表明, 分形维数 $D$ 和分形粗粘度 $G^{*}$ 是影响结合面法向接触阻尼损耗因子和法向接触阻尼系数的最主要参数。在塑性指 数 $\phi$ 不变, $D$ 小于拐点值 $(D=1.56)$ 时, 法向接触阻尼系数随着 $G^{*}$ 的增大而增大; 当 $D$ 超过拐点值时, $G^{*}$ 越大, 法向接触阻尼 系数减小。当 $G^{*}$ 不变, $D<1.66$ 时, $\phi$ 越大, 法向接触阻尼系数越小; 当 $D>1.66$ 时, 法向接触阻尼系数随 $\phi$ 的变化很小。利 用线轨滑台模态试验验证所建模型的准确与可靠性。
\end{abstract}

关键词: 分形理论; 弹塑性接触模型；法向接触阻尼；阻尼损耗因子；线轨滑台

中图分类号: TH113

\section{Fractal Model for Normal Contact Damping of Joint Surface Considering Elastoplastic Phase}

\author{
CHEN Yonghui ZHANG Xueliang WEN Shuhua LAN Guosheng
}

(College of Mechanical Engineering, Taiyuan University of Science and Technology, Taiyuan 030024)

\begin{abstract}
When modeling the normal contact damping of the joint surface, it is incomplete to consider only the elastic energy of the asperity in the perfectly elastic deformation phase and the loss energy in the fully plastic deformation phase, because there is the elastic-plastic deformation phase. The normal contact load of the contacting asperity in the elastoplastic deformation phase is "separated" into the elastic contact load and the plastic contact load, and the elastic and loss energy of the contacting asperity are obtained. The normal contact dynamic model of the joint surface is equivalent to spring and viscous damper, and the analytic model of damping loss factor and normal contact damping of the joint surface is established, and the dimensionless processing is carried out. The results reveal that fractal dimension $D$ and fractal roughness $G^{*}$ are the important parameters affecting the damping loss factor and the normal contact damping coefficient. When the plastic index $\phi$ is constant and $D$ is less than the inflection point value $(D=1.56)$, the normal contact damping coefficient increases with the increase of; when $D$ exceeds the inflection point value, it decreases with the increase of $G^{*}$. When $G^{*}$ remains unchanged and $D$ is less than 1.66 , the greater the plastic index, the smaller the normal contact damping coefficient; when $D$ is greater than 1.66 , the change of the normal contact damping coefficient with $\phi$ is very small. The accuracy and reliability of the model are verified by the results of experimental modal analysis on line rail sliding table.
\end{abstract}

Key words: fractal theory; elastic-plastic contact model; normal contact damping; damping loss factor; line rail sliding table

\section{0 前言}

机械结构中存在着大量的结合面, 并且结合面

* 国家自然科学基金(51275328)、山西省自然科学基金(201601D011062) 和山西省 “1331” 工程重点学科建设资助项目。20181025 收到初稿, 20190513 收到修改稿
的接触参数 (如接触刚度和接触阻尼), 对整体的静 态特性、动态特性都有重大影响 ${ }^{[1-3]}$ 。因此, 近些年 国内外学者对结合面接触刚度和接触阻尼建模研究 变得较为活跃, 成果也较多。

结合面接触模型的研究, 从最早的 Hertz 接触 理论, 到后来 Greenwood 和 Williamson 的 GW 模型, 以及其他学者建立的适用于极大载荷下的塑性接触 
模型。这些模型均忽略了弹性变形和塑性变形之间 的弹塑性变形区间。近年来, 大多数学者从微接触 角度建立微凸体的接触模型, 文献[4-9]对于弹塑性 变形区间的拟合模型进行了研究, 其中模型有: CEB 模型、 ZMC 模型、 $\mathrm{KE}$ 模型、三次样条函数对 ZMC 模型的改进、Hermit 多项式插值模型、低阶椭圆曲 线插值方法拟合建模等。文献[10-15]在进行结合面 法向接触刚度建模时, 大多采用 $\mathrm{KE}$ 模型、多项式 插值模型等推导了结合面三阶段的法向接触刚度和 法向接触载荷之间的关系。但上述三阶段接触建模 存在不连续或不合理问题, 故文献[16]建立了基于 指数函数的弹塑性阶段模型和结合面法向接触刚度 模型。早期法向接触阻尼的研究大多从宏观角度对 其进行试验研究 ${ }^{[17-19]}$, 间接识别特定结合面的接触 阻尼, 但是此方法不仅试验量大, 而且通用性、遍 历性和可靠性难保证。文献[14-15、20-21]研究了结 合面法向接触阻尼建模, 给出了与结合部件的基体 质量有关的接触阻尼解析模型。

现有结合面法向接触阻尼建模研究均未考虑微 凸体接触时的弹塑性阶段, 主要原因是无法将弹塑 性阶段的接触载荷 “分离” 为弹性载荷和塑性载荷。 本文基于文献[16]中弹塑性阶段的接触载荷模型, 建立了微凸体法向接触阻尼分形模型以及结合面的 法向接触阻尼分形模型, 并对模型进行了仿真分析。 同时, 利用法向接触模型计算的参数对线轨滑台进 行了理论模态分析, 并进行了试验模态分析验证。

\section{1 微凸体法向接触阻尼的分形模型}

文献[16]沿用文献[11]中 $\mathrm{GW}$ 模型的假设, 得到 微凸体在完全弹性阶段(即 $0 \leqslant \delta \leqslant \delta_{\mathrm{c}}$ 时)、弹塑性阶 段 (即 $\delta_{\mathrm{c}} \leqslant \delta \leqslant 110 \delta_{\mathrm{c}}$ 时) 以及完全塑性阶段 (即 $\delta \geqslant 110 \delta_{c}$ 时)的接触载荷 $F_{\mathrm{e}}(\delta) 、 F_{\mathrm{ep}}(\delta)$ 和 $F_{\mathrm{p}}(\delta)$, 分别为

$$
\begin{gathered}
F_{\mathrm{e}}(\delta)=\frac{4}{3} E R^{\frac{1}{2}} \delta^{\frac{3}{2}} \\
F_{\mathrm{ep}}(\delta)=\frac{4}{3} E R^{\frac{1}{2}} \delta^{\frac{3}{2}}+\left(2 \pi R \delta H-\frac{4}{3} E R^{\frac{1}{2}} \delta^{\frac{3}{2}}\right) \times \\
{\left[\frac{2\left(\delta-\delta_{\mathrm{c}}\right)}{109 \delta_{\mathrm{c}}}-\left(\frac{\delta-\delta_{\mathrm{c}}}{109 \delta_{\mathrm{c}}}\right)^{2}\right]} \\
F_{\mathrm{p}}(\delta)=2 \pi R \delta H
\end{gathered}
$$

式中, $E$ 为复合弹性模量 ${ }^{[22]}$ (微凸体与一刚性平面接 触), $E=E_{1} /\left(1-\mu^{2}\right), E_{1}$ 为微凸体接触材料的弹性 模量, $\mu$ 为泊松比; $\delta_{\mathrm{c}}$ 为临界接触变形量, $\delta_{\mathrm{c}}=(\pi K H / 2 E)^{2} R, K=0.454+0.41 \mu ; H$ 为硬度。
微凸体曲率半径 $R$ 和接触变形量 $\delta$ 分别为

$$
\begin{gathered}
R=\frac{a^{\frac{D}{2}}}{2 \pi \mathrm{g}_{1}(D)} \\
\delta=g_{1}(D) a^{\frac{2-D}{2}}
\end{gathered}
$$

式中, $g_{1}(D)=2^{3-D} \pi^{(D-2) / 2} G^{D-1}(\ln \beta)^{1 / 2}, a$ 为微接触 点的面积。由式(5)可得

$$
\mathrm{d} \delta=\frac{2-D}{2} g_{1}(D) a^{\frac{-D}{2}} \mathrm{~d} a
$$

因此微凸体在弹性接触阶段的弹性势能为

$$
w_{\mathrm{e}}(\delta)=\int_{0}^{\delta} F_{\mathrm{e}}(\delta) \mathrm{d} \delta=\int_{0}^{\delta} \frac{4}{3} E R^{\frac{1}{2}} \delta^{\frac{3}{2}} \mathrm{~d} \delta
$$

将式(4) (6)分别代入式(7)中, 可得

$$
w_{\mathrm{e}}(a)=\frac{4(2-D) E \mathrm{~g}_{1}^{2}(D)}{3 \sqrt{2 \pi}(5-2 D)} a^{\frac{5-2 D}{2}}
$$

同理可得微凸体在塑性接触阶段损耗能为

$$
w_{\mathrm{p}}(a)=\int_{0}^{\delta} F_{\mathrm{p}}(\delta) \mathrm{d} \delta=\frac{(2-D) H \mathrm{~g}_{1}(D)}{4-D} a^{\frac{4-D}{2}}
$$

由文献 [16] 可知, 弹塑性变形阶段 (即 $\delta_{\mathrm{c}} \leqslant \delta \leqslant 110 \delta_{\mathrm{c}}$ 时), 既存在弹性变形区域又存在塑 性变形区域。而之前文献在计算结合面法向接触阻 尼时, 均未考虑微凸体弹塑性接触阶段的法向接触 载荷产生的弹性势能和损耗能, 这样的计算结果是 不合理的。本文中基于文献[16]中的微凸体弹塑性 阶段的法向接触载荷, 它是由弹性阶段和塑性阶段 两部分的法向接触载荷拟合而成, 是可以明确 “分 离” 开来的。因此, 由式(2)可将 $F_{\mathrm{ep}}(\delta)$ 重新写成弹 性接触载荷 $F_{\mathrm{ep} 1}(\delta)$ 和塑性接触载荷 $F_{\mathrm{ep} 2}(\delta)$ 之和

$$
F_{\text {ep }}(\delta)=F_{\text {ep } 1}(\delta)+F_{\text {ep } 2}(\delta) \quad \delta_{\mathrm{c}} \leqslant \delta \leqslant 110 \delta_{\mathrm{c}}
$$

式中, $F_{\mathrm{ep} 1}(\delta)=\frac{4}{3} E R^{\frac{1}{2}} \delta^{\frac{3}{2}}\left[1-\frac{2\left(\delta-\delta_{\mathrm{c}}\right)}{109 \delta_{\mathrm{c}}}-\left(\frac{\delta-\delta_{\mathrm{c}}}{109 \delta_{\mathrm{c}}}\right)^{2}\right]$, $F_{\mathrm{ep} 2}(\delta)=2 \pi R \delta H\left[\frac{2\left(\delta-\delta_{\mathrm{c}}\right)}{109 \delta_{\mathrm{c}}}-\left(\frac{\delta-\delta_{\mathrm{c}}}{109 \delta_{\mathrm{c}}}\right)^{2}\right]$ 。而且, 临 界接触变形量 $\delta_{\mathrm{c}}$ 为

$$
\delta_{\mathrm{c}}=\left(\frac{\pi K H}{2 E}\right)^{2} R=\left(\frac{\pi K H}{2 E}\right)^{2} \frac{a^{\frac{D}{2}}}{2 \pi g_{1}(D)}
$$

将式(5)代入式(11), 可得

$$
\frac{\delta}{\delta_{\mathrm{c}}}=\frac{8 g_{1}^{2}(D)}{\pi}\left(\frac{E}{K H}\right)^{2} \frac{1}{a^{D-1}}
$$

并且, 临界接触面积 $a_{\mathrm{c}}$ 为

$$
a_{\mathrm{c}}=\left[2^{9-2 D} \pi^{D-3} G^{2 D-2} \ln \beta\left(\frac{E}{K H}\right)^{2}\right]^{\frac{1}{D-1}}=
$$




$$
\left[\frac{8 g_{1}^{2}(D)}{\pi}\left(\frac{E}{K H}\right)^{2}\right]^{\frac{1}{D-1}}
$$

即

$$
a_{\mathrm{c}}^{D-1}=\frac{8 g_{1}^{2}(D)}{\pi}\left(\frac{E}{K H}\right)^{2}
$$

所以, 由式(12)和式(13), 可得接触变形量和接 触面积之间的关系为

$$
\frac{\delta}{\delta_{\mathrm{c}}}=\left(\frac{a_{\mathrm{c}}}{a}\right)^{D-1}=\left(\frac{a}{a_{\mathrm{c}}}\right)^{1-D}
$$

将式(14)代入式(10)中的 $F_{\mathrm{ep} 1}(\delta)$ 和 $F_{\mathrm{ep} 2}(\delta)$, 并 且分别计算, 得到微凸体在弹塑性阶段的弹性势能 $w_{\text {ep } 1}$ 和损耗能 $w_{\text {ep2 } 2}$ 分别为

$$
\begin{aligned}
& w_{\text {ep } 1}=\int_{0}^{\delta} F_{\text {ep } 1}(\delta) \mathrm{d} \delta= \\
& \int_{0}^{a} \frac{2(2-D) E g_{1}^{2}(D)}{3 \sqrt{2 \pi}} a^{\frac{3-2 D}{2}} \times \\
& {\left[1-\frac{2\left(a^{1-D}-a_{\mathrm{c}}^{1-D}\right)}{109 a_{\mathrm{c}}^{1-D}}-\left(\frac{a^{1-D}-a_{\mathrm{c}}^{1-D}}{109 a_{\mathrm{c}}^{1-D}}\right)^{2}\right] \mathrm{d} a=} \\
& \frac{4(2-D) E g_{1}^{2}(D)}{3 \sqrt{2 \pi}}\left[\left(\frac{110}{109}\right)^{2} \frac{1}{5-2 D} a^{\frac{5-2 D}{2}}-\right. \\
& \frac{220}{109^{2}(7-4 D) a_{\mathrm{c}}^{1-D}} a^{\frac{7-4 D}{2}}+ \\
& \left.\frac{1}{109^{2}(9-6 D) a_{\mathrm{c}}^{2(1-D)}} a^{\frac{9-6 D}{2}}\right] \\
& w_{\mathrm{ep} 2}=\int_{0}^{\delta} F_{\mathrm{ep} 2}(\delta) \mathrm{d} \delta= \\
& \int_{0}^{a} \frac{(2-D) H g_{1}(D)}{2} a^{\frac{2-D}{2}} \times \\
& {\left[\frac{2\left(a^{1-D}-a_{\mathrm{c}}^{1-D}\right)}{109 a_{\mathrm{c}}^{1-D}}-\left(\frac{a^{1-D}-a_{\mathrm{c}}^{1-D}}{109 a_{\mathrm{c}}^{1-D}}\right)^{2}\right] \mathrm{d} a=} \\
& (2-D) H g_{1}(D)\left[\frac{-219}{109^{2}(4-D)} a^{\frac{4-D}{2}}-\right. \\
& \left.\frac{220}{109^{2}(6-3 D) a_{\mathrm{c}}^{1-D}} a^{\frac{6-3 D}{2}}+\frac{1}{109^{2}(8-5 D) a_{\mathrm{c}}^{2(1-D)}} a^{\frac{8-5 D}{2}}\right]
\end{aligned}
$$

\section{2 结合面法向接触阻尼分形模型}

由文献[23]可知, 结合面微接触点的面积分布 密度函数为

$$
n(a)=\frac{D}{2} a_{\mathrm{L}}^{\frac{D}{2}} a^{-\left(\frac{D}{2}+1\right)} \quad 0<a \leqslant a_{\mathrm{L}}
$$

式中, $a_{\mathrm{L}}$ 为最大微接触点的面积。

结合面的真实接触面积 $A_{\mathrm{r}}$ 为

$$
A_{\mathrm{r}}=\int_{0}^{a_{\mathrm{L}}} \operatorname{an}(a) \mathrm{d} a=\frac{D}{2-D} a_{\mathrm{L}}
$$

即

$$
a_{\mathrm{L}}=\frac{2-D}{D} A_{\mathrm{r}}
$$

结合面总的弹性势能为

$$
\begin{gathered}
W_{\mathrm{e}}=\int_{a_{\mathrm{c}}}^{a_{\mathrm{L}}} w_{\mathrm{e}}(a) n(a) \mathrm{d} a+\int_{a_{\mathrm{c} 1}}^{a_{\mathrm{c}}} w_{\mathrm{e} 1}(a) n(a) \mathrm{d} a= \\
W_{\mathrm{e} 1}+W_{\mathrm{e} 2}
\end{gathered}
$$

将式(8)、式(15)和式(17)代入式(19)中，分别得到 $W_{\mathrm{e} 1} 、 W_{\mathrm{e} 2}$

$$
\begin{aligned}
& W_{\mathrm{e} 1}=\int_{a_{\mathrm{c}}}^{a_{\mathrm{L}}} \frac{4(2-D) E g_{1}^{2}(D)}{3 \sqrt{2 \pi}(5-2 D)} a^{\frac{5-2 D}{2}} \frac{D}{2} a_{\mathrm{L}}^{\frac{D}{2}} a^{-\left(\frac{D}{2}+1\right)} \mathrm{d} a= \\
& \frac{4(2-D) D E g_{1}^{2}(D)}{3 \sqrt{2 \pi}(5-2 D)(5-3 D)} a_{\mathrm{L}}^{\frac{D}{2}}\left(a_{\mathrm{L}}^{\frac{5-3 D}{2}}-a_{\mathrm{c}}^{\frac{5-3 D}{2}}\right) \\
& W_{\mathrm{e} 2}=\int_{a_{\mathrm{c} 1}}^{a_{\mathrm{c}}} \frac{4(2-D) E g_{1}^{2}(D)}{3 \sqrt{2 \pi}}\left[\left(\frac{110}{109}\right)^{2} \frac{1}{5-2 D} a^{\frac{5-2 D}{2}}-\right. \\
& \left.\frac{220}{109^{2}(7-4 D) a_{\mathrm{c}}^{1-D}} a^{\frac{7-4 D}{2}}+\frac{1}{109^{2}(9-6 D) a_{\mathrm{c}}^{2(1-D)}} a^{\frac{9-6 D}{2}}\right] \times \\
& \frac{D}{2} a_{\mathrm{L}}^{\frac{D}{2}} a^{-\left(\frac{D}{2}+1\right)} \mathrm{d} a= \\
& \frac{4(2-D) D E g_{1}^{2}(D)}{3 \sqrt{2 \pi}} a_{\mathrm{L}}^{\frac{D}{2}}\left[\left(\frac{110}{109}\right)^{2} \frac{a_{\mathrm{c}}^{\frac{5-3 D}{2}}-a_{\mathrm{c} 1}^{\frac{5-3 D}{2}}}{(5-2 D)(5-3 D)}-\right. \\
& \frac{220\left(a_{\mathrm{c}}^{\frac{7-5 D}{2}}-a_{\mathrm{c} 1}^{\frac{7-5 D}{2}}\right)}{109^{2}(7-4 D)(7-5 D) a_{\mathrm{c}}^{1-D}}+ \\
& \left.\frac{a_{\mathrm{c}}^{\frac{9-7 D}{2}}-a_{\mathrm{c} 1}^{\frac{9-7 D}{2}}}{109^{2}(9-6 D)(9-7 D) a_{\mathrm{c}}^{2(1-D)}}\right]
\end{aligned}
$$

由文献[11]可知, 微凸体从弹塑性接触阶段到完 全塑性接触阶段的临界接触面积 $a_{\mathrm{c} 1}$ 与 $a_{\mathrm{c}}$ 的关系为

$$
a_{\mathrm{c} 1}=\left(\frac{1}{110}\right)^{\frac{1}{D-1}} a_{\mathrm{c}}
$$

结合面总的损耗能 $W_{\mathrm{p}}$ 为

$$
\begin{gathered}
W_{\mathrm{p}}=\int_{0}^{a_{\mathrm{c} 1}} w_{\mathrm{p}}(a) n(a) \mathrm{d} a+\int_{a_{\mathrm{c} 1}}^{a_{\mathrm{c}}} w_{\mathrm{ep} 2}(a) n(a) \mathrm{d} a= \\
W_{\mathrm{p} 1}+W_{\mathrm{p} 2}
\end{gathered}
$$

将式(9)、式(16)和式(17)代入式(23)中，分别得到 $W_{\mathrm{p} 1} 、 W_{\mathrm{p} 2}$ 


$$
\begin{gathered}
W_{\mathrm{p} 1}=\int_{0}^{a_{\mathrm{cl}}} \frac{(2-D) H g_{1}(D)}{4-D} a^{\frac{4-D}{2}} \frac{D}{2} a_{\mathrm{L}}^{\frac{D}{2}} a^{-\left(\frac{D}{2}+1\right)} \mathrm{d} a= \\
\frac{D H g_{1}(D)}{2(4-D)} a_{\mathrm{L}}^{\frac{D}{2}} a_{\mathrm{c} 1}^{2-D} \\
W_{\mathrm{p} 2}=\int_{a_{\mathrm{c} 1}}^{a_{\mathrm{c}}}(2-D) H g_{1}(D)\left[\frac{-219}{109^{2}(4-D)} a^{\frac{4-D}{2}}+\right. \\
\left.\frac{220}{109^{2}(6-3 D) a_{\mathrm{c}}^{1-D}} a^{\frac{6-3 D}{2}}-\frac{1}{109^{2}(8-5 D) a_{\mathrm{c}}^{2(1-D)}} a^{\frac{8-5 D}{2}}\right] \times \\
\frac{(2-D) D H g_{1}(D)}{2} a_{\mathrm{L}}^{\frac{D}{2}} a^{\frac{D}{2}}\left[\frac{-219\left(a_{\mathrm{c}}^{2}+1\right)}{\mathrm{d} a=}-a_{\mathrm{c} 1}^{2-D}\right) \\
\frac{220\left(a_{\mathrm{c}}^{3-2 D}-a_{\mathrm{c} 1}^{3-2 D}\right)}{109^{2}(4-D)(2-D)}+ \\
\left.109^{2}(6-3 D)(3-2 D) a_{\mathrm{c}}^{1-D}-\frac{a_{\mathrm{c}}^{4-3 D}-a_{\mathrm{c} 1}^{4-3 D}}{109^{2}(8-5 D)(4-3 D) a_{\mathrm{c}}^{2(1-D)}}\right]
\end{gathered}
$$

由式(19)和式(23)(并将式(20)、式(21)、式(24) 和式(25)代入)可得结合面的阻尼损耗因子 $\eta$ 为

$$
\begin{gathered}
\eta=\frac{W_{\mathrm{p}}}{W_{\mathrm{e}}}=\frac{3 \sqrt{2 \pi} H}{8 E \mathrm{~g}_{1}(D)}\left[\frac{a_{\mathrm{c} 1}^{2-D}}{(4-D)(2-D)}-\right. \\
\frac{219\left(a_{\mathrm{c}}^{2-D}-a_{\mathrm{c} 1}^{2-D}\right)}{109^{2}(4-D)(2-D)}+ \\
\frac{220\left(a_{\mathrm{c}}^{3-2 D}-a_{\mathrm{c} 1}^{3-2 D}\right)}{109^{2}(6-3 D)(3-2 D) a_{\mathrm{c}}^{1-D}}- \\
\left.\frac{a_{\mathrm{c}}^{4-3 D}-a_{\mathrm{c} 1}^{4-3 D}}{109^{2}(8-5 D)(4-3 D) a_{\mathrm{c}}^{2(1-D)}}\right] \div \\
\left.\frac{a_{\mathrm{L}}^{\frac{5-3 D}{2}}}{(5-2 D)(5-3 D)}+\frac{a_{\mathrm{c}}^{\frac{5-3 D}{2}}}{\left(\frac{110}{109}\right)^{2}\left(a_{\mathrm{c}}^{\frac{5-3 D}{2}}-a_{\mathrm{c} 1}^{\frac{5-3 D}{2}}\right.}\right) \\
\frac{(5-2 D)(5-3 D)}{220} \cdot \frac{a_{\mathrm{c}}^{\frac{7-5 D}{2}}-a_{\mathrm{c} 1}^{\frac{7-5 D}{2}}}{(7-4 D)(7-5 D) a_{\mathrm{c}}^{1-D}}+ \\
\left.\frac{a_{\mathrm{c}}^{\frac{9-7 D}{2}}-a_{\mathrm{c} 1}^{\frac{9-7 D}{2}}}{109^{2}(9-6 D)(9-7 D) a_{\mathrm{c}}^{2(1-D)}}\right]
\end{gathered}
$$

结合面基体质量和结合面质量不是一个概念, 基体质量不属于结合面的内在参数, 而结合面质量 属于结合面的内在参数。在建立结合面接触模型时 考虑基体质量, 将结合面法向动力学模型简化成质 量(基体质量)-阻尼-弹簧的单自由度系统是将基体
质量和结合面作为一个系统来考虑, 不具有普适性。 本文不考虑基体质量，而且结合面本身的质量微乎 其微, 因此忽略结合面本身的质量, 将结合面法向 接触模型等效为弹簧和黏性阻尼器 ${ }^{[1,24-25]}$ 。结合面 法向动力学模型如图 1 所示。

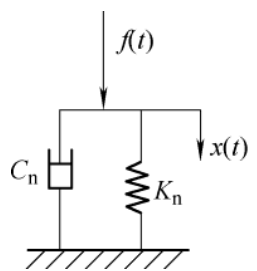

图 1 结合面接触动力学简化模型

由图 1 可得, 动力学方程为

$$
f(t)=K_{\mathrm{n}} x(t)+C_{\mathrm{n}} \dot{x}(t)
$$

激励力 $f(t)=F_{\mathrm{n}} \cos \omega t$, 则 $x(t)=X \cos \left(\omega-\varphi_{\mathrm{n}}\right)$, 因 此, $\eta=W_{\mathrm{p}} / W_{\mathrm{e}}=\left|f_{\mathrm{c}} x(t)\right| /\left|f_{\mathrm{k}} x(t)\right|=C_{\mathrm{n}} \omega / K_{\mathrm{n}}$, 即

$$
C_{\mathrm{n}}=\frac{\eta K_{\mathrm{n}}}{\omega}
$$

对式(28)进行量纲一化处理, 得到结合面量纲一法 向接触阻尼 $C_{\mathrm{n}}^{*}$ 为

$$
C_{\mathrm{n}}^{*}=\frac{C_{\mathrm{n}} \omega}{H \sqrt{A_{\mathrm{a}}}}=\frac{K_{\mathrm{n}}}{E \sqrt{A_{\mathrm{a}}}} \cdot \frac{\eta E}{H}=K_{\mathrm{n}}^{*} \frac{\eta}{\phi}
$$

其中, 引用文献[16]中量纲一法向接触刚度 $K_{\mathrm{n}}^{*}$ 为

$$
K_{\mathrm{n}}^{*}=\frac{4(3-D) D^{\frac{2-D}{2}} A_{\mathrm{r}}^{* \frac{D}{2}}\left[\left(\frac{2-D}{D} A_{\mathrm{r}}^{*}\right)^{\frac{1-D}{2}}-a_{\mathrm{c}}^{* \frac{1-D}{2}}\right]}{3 \sqrt{2 \pi}(1-D)(2-D)^{\frac{2-D}{2}}}+
$$

$$
\frac{4 \times 110^{2}(3-D) D^{\frac{2-D}{2}} A_{\mathrm{r}}^{* \frac{D}{2}}\left[1-\left(\frac{1}{110}\right)^{-\frac{1}{2}}\right]}{3 \times 109^{2} \sqrt{2 \pi}(1-D)(2-D)^{\frac{2-D}{2}}} a_{\mathrm{c}}^{* \frac{1-D}{2}}+
$$

$$
\frac{110(4-D) D^{\frac{2-D}{2}} \phi A_{\mathrm{r}}^{* \frac{D}{2}}\left[1-\left(\frac{1}{110}\right)^{\frac{2-D}{2(D-1)}}\right]}{109^{2} \times 2^{2-D}(2-D)^{\frac{4-D}{2}} \pi^{\frac{D-2}{2}} G^{* D-1}(\ln \beta)^{\frac{1}{2}}}+
$$

$$
\frac{219 D^{\frac{2-D}{2}} \phi A_{\mathrm{r}}^{* \frac{D}{2}}\left(\frac{D-1}{D} \ln 110\right)}{109^{2} \times 2^{3-D}(2-D)^{\frac{2-D}{2}} \pi^{\frac{D-2}{2}} G^{* D-1}(\ln \beta)^{\frac{1}{2}}}-
$$

$$
\frac{(3-D) D^{\frac{2-D}{2}} \phi A_{\mathrm{r}}^{* \frac{D}{2}}\left[1-\left(\frac{1}{110}\right)^{\frac{2-D}{D-1}}\right]}{109^{2} \times 2^{3-D}(2-D)^{\frac{4-D}{2}} \pi^{\frac{D-2}{2}} G^{* D-1}(\ln \beta)^{\frac{1}{2}}}-
$$




$$
\begin{gathered}
\frac{880(5-2 D) D^{\frac{2-D}{2}} A_{\mathrm{r}}^{* \frac{D}{2}}\left[1-\left(\frac{1}{110}\right)^{\left.\frac{3-2 D}{2(D-1)}\right]} a_{\mathrm{c}}^{* \frac{1-D}{2}}-\right.}{3 \times 109^{2} \sqrt{2 \pi}(3-2 D)(2-D)^{\frac{2-D}{2}}} \\
\frac{4(7-3 D) D^{\frac{2-D}{2}} A_{\mathrm{r}}^{* \frac{D}{2}}\left[1-\left(\frac{1}{110}\right)^{\frac{5-3 D}{2(D-1)}}\right]}{3 \times 109^{2} \sqrt{2 \pi}(5-3 D)(2-D)^{\frac{2-D}{2}}} a_{\mathrm{c}}^{* \frac{1-D}{2}}
\end{gathered}
$$

式中, $K_{\mathrm{n}}^{*}=\frac{K_{\mathrm{n}}}{E \sqrt{A_{\mathrm{a}}}}, A_{\mathrm{r}}^{*}=\frac{A_{\mathrm{r}}}{A_{\mathrm{a}}}, a_{\mathrm{c}}^{*}=\frac{a_{\mathrm{c}}}{A_{\mathrm{a}}}, G^{*}=\frac{G}{\sqrt{A_{\mathrm{a}}}}$, $\phi=\frac{H}{E}$ 。

将式(18)和式(22)代入式(26), 并且结合式(29)可 以得到用量纲一的量来表示的阻尼损耗因子 $\eta$, 即

$$
\eta=\frac{3 G^{* 1-D} a_{\mathrm{c}}^{* \frac{D-1}{2}}}{2^{\frac{11-2 D}{2}} \pi^{\frac{D-3}{2}} \phi(\ln \beta)^{\frac{1}{2}}}\left\{\frac{\left[\left(1+\frac{219}{109^{2}}\right)\left(\frac{1}{110}\right)^{\frac{2-D}{D-1}}-\frac{219}{109^{2}}\right]}{(4-D)(2-D)}+\right.
$$

$$
\left.\frac{220\left[1-\left(\frac{1}{110}\right)^{\frac{3-2 D}{D-1}}\right]}{109^{2}(6-3 D)(3-2 D)}-\frac{\left[1-\left(\frac{1}{110}\right)^{\frac{4-3 D}{D-1}}\right]}{109^{2}(8-5 D)(4-3 D)}\right\} \div
$$

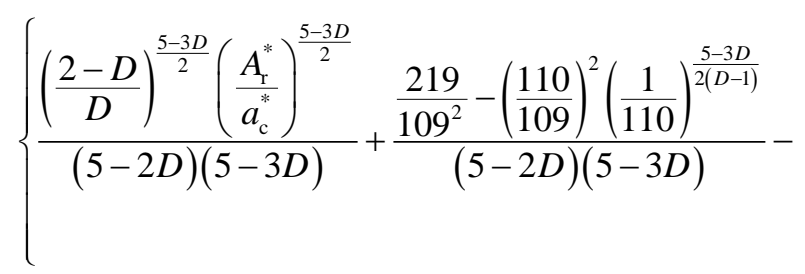

$$
\left.\frac{220\left[1-\left(\frac{1}{110}\right)^{\frac{7-5 D}{2(D-1)}}\right]}{109^{2}(7-4 D)(7-5 D)}+\frac{\left[1-\left(\frac{1}{110}\right)^{\frac{9-7 D}{2(D-1)}}\right]}{109^{2}(9-6 D)(9-7 D)}\right\}
$$

\section{3 结合面法向接触阻尼仿真与分析}

从式(30)、式(31)和式(29)可以看出, 量纲一法 向接触刚度 $K_{\mathrm{n}}^{*}$ 、阻尼损耗因子 $\eta$ 和量纲一法向接触 阻尼 $C_{\mathrm{n}}^{*}$ 是量纲一真实接触面积 $A_{\mathrm{r}}^{*}$ 、分形维数 $D$ 、 量纲一分形粗糙度参数 $G^{*}$ 和塑性指数 $\phi$ 等变量的 函数。仿真分析时, 各参数的取值范围分别为: $0 \leqslant A_{\mathrm{r}}^{*} \leqslant 0.15 ; 1.01 \leqslant D \leqslant 1.99$ ，但是因计算结合 面法向接触载荷、法向接触刚度、阻尼损耗因子以 及法向接触阻尼时, 当 $D$ 为特殊值时, 会出现积分 结果中分母为 0 的情况, 通常做法是重新给出特殊 $D$ 时的相应公式, 而在本文中, 没有给出特殊表达, 而是用统一的公式来代替，并得到了统一的阻尼损 耗因子和量纲一接触阻尼。因此, 为了避开 $D=3 / 2$ 、 $5 / 3 、 7 / 4 、 7 / 5 、 9 / 7$ 等特殊值, 仿真分析时, $D$ 的取 值为: $1.16 、 1.26 、 \cdots 、 1.96 ; G^{*}$ 的取值为 $10^{-9} 、 10^{-10}$ 、 $10^{-11} ; \phi$ 的取值为 $0.7 、 1.5 、 2.5$ 。利用各个变量的 数据对式(31)和式(29)进行仿真, 分别得到相应的结 果, 如图 2 6 所示(说明: 由于分析时纵坐标相差 较大, 为了更好地描述图形, 图 2 6 中的纵坐标均 采用对数坐标, 即纵坐标为 $\lg \eta$ 或 $\lg \left(C_{\mathrm{n}}^{*}\right)$ 。)。

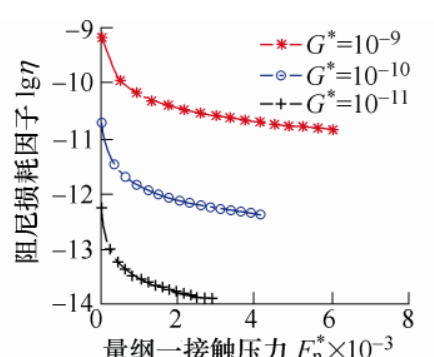

(a) $D=1.16$

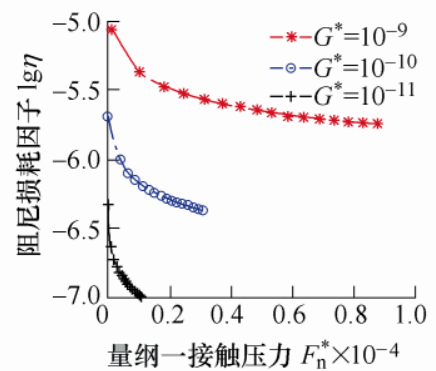

(d) $D=1.46$

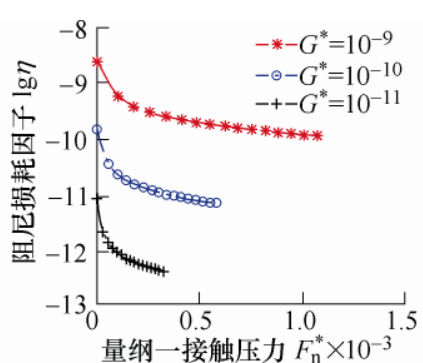

(b) $D=1.26$

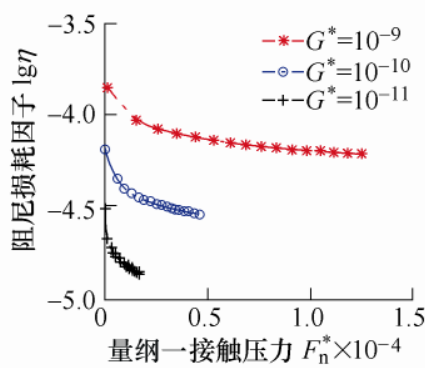

(e) $D=1.56$

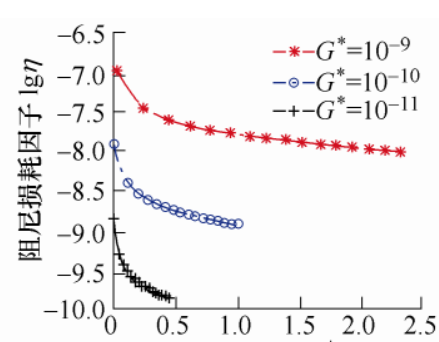

量纲一接触压力 $F_{\mathrm{n}}^{*} \times 10^{-4}$

(c) $D=1.36$

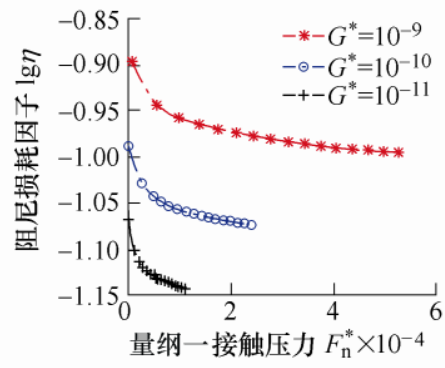

(f) $D=1.66$ 


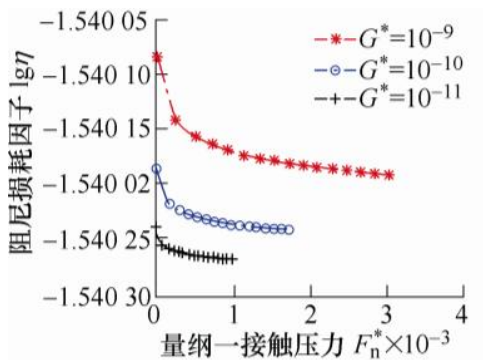

(g) $D=1.76$

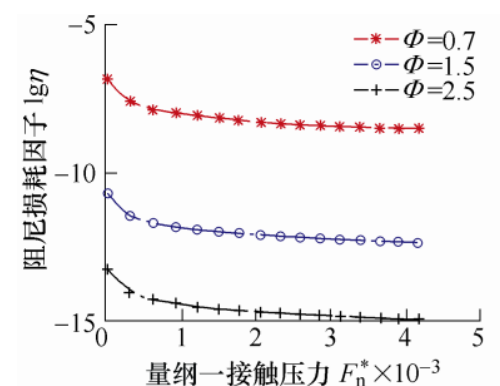

(a) $D=1.16$

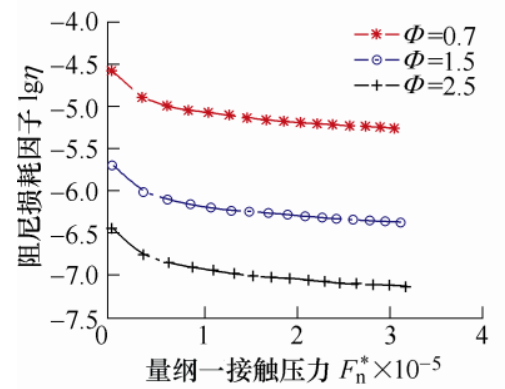

(d) $D=1.46$

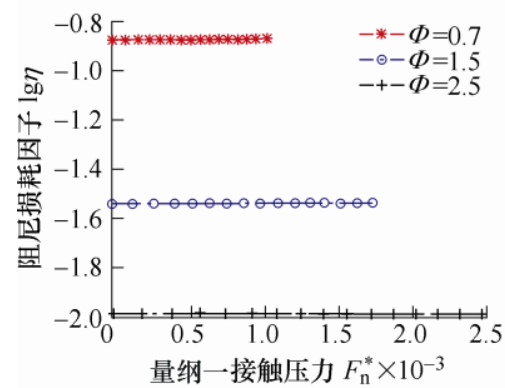

(g) $D=1.76$

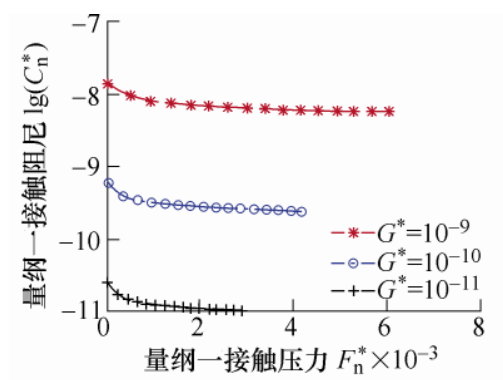

(a) $D=1.16$

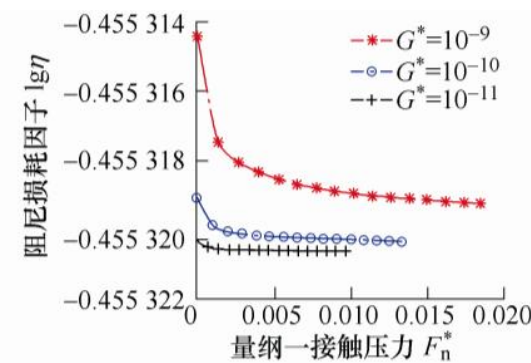

(h) $D=1.86$

图 $2 \lg \eta$ 随着 $F_{\mathrm{n}}{ }^{*}$ 的变化 $(\phi=1.5)$

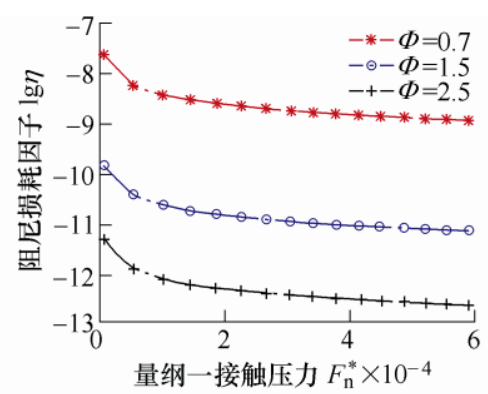

(b) $D=1.26$

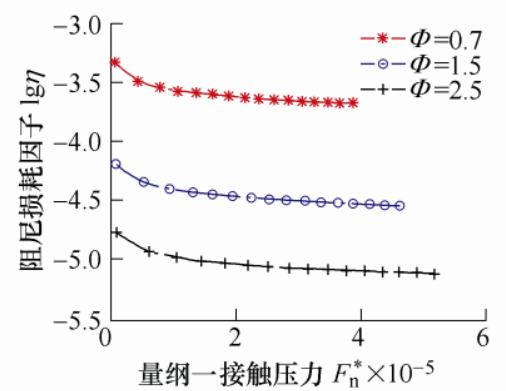

(e) $D=1.56$

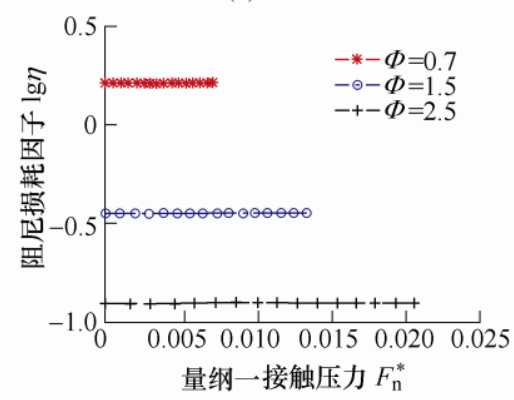

(h) $D=1.86$

图 $3 \lg \eta$ 随着 $F_{\mathrm{n}}^{*}$ 的变化 $\left(G^{*}=10^{-10}\right)$

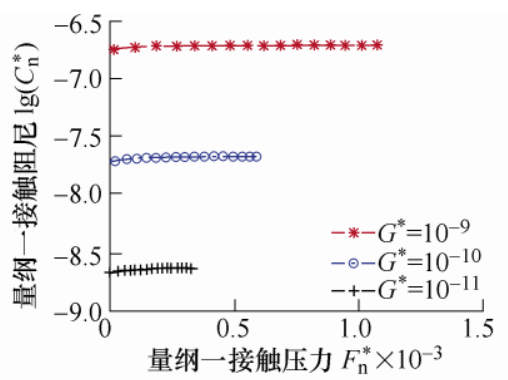

(b) $D=1.26$

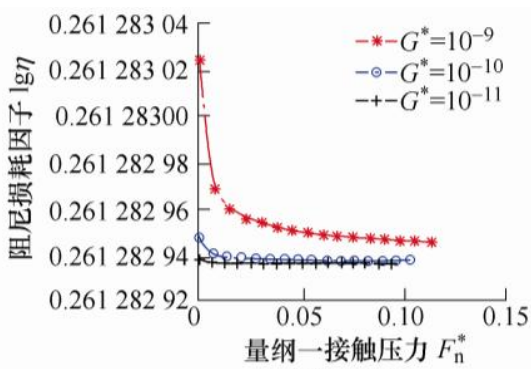

(i) $D=1.96$

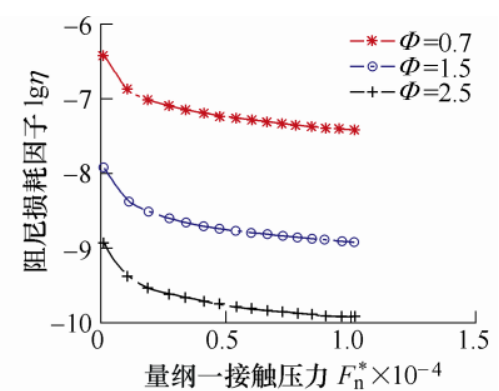

(c) $D=1.36$

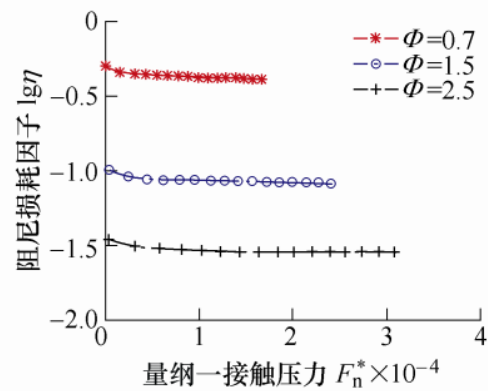

(f) $D=1.66$

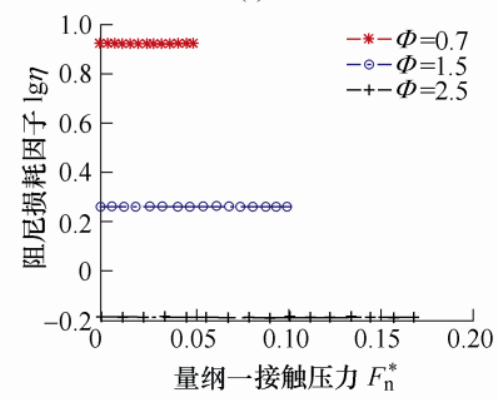

(i) $D=1.96$

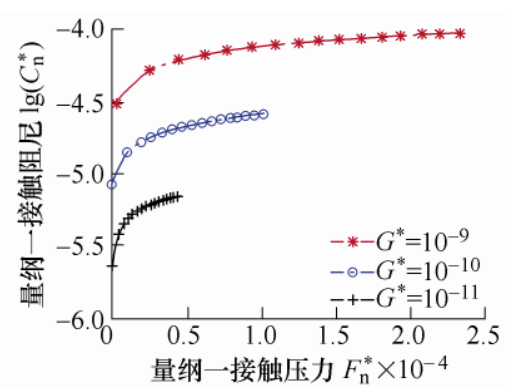

(c) $D=1.36$ 


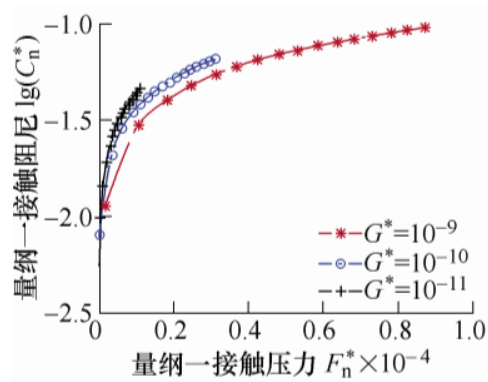

(d) $D=1.46$

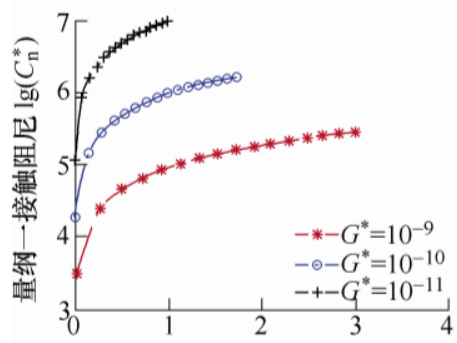

量纲一接触压力 $F_{\mathrm{n}}^{*} \times 10^{-3}$

(g) $D=1.76$

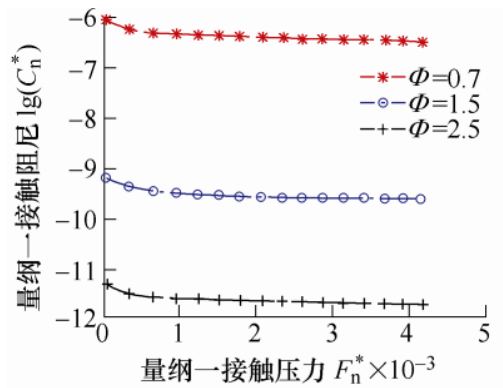

(a) $D=1.16$

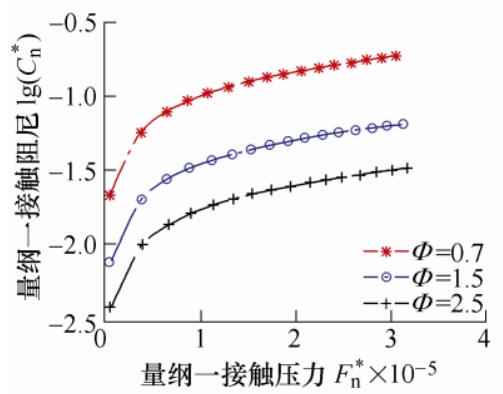

(d) $D=1.46$

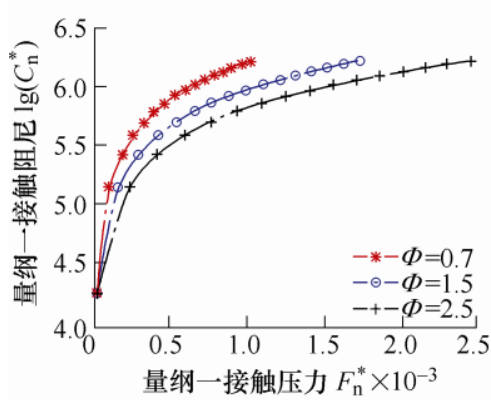

(g) $D=1.76$

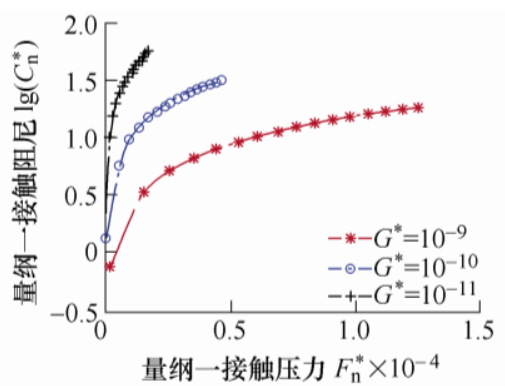

(e) $D=1.56$

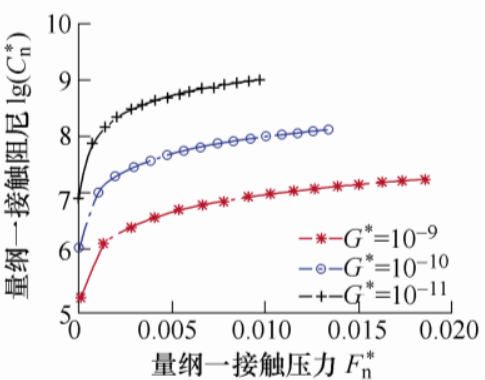

(h) $D=1.86$

图 $4 \lg \left(C_{\mathrm{n}}^{*}\right)$ 随着 $F_{\mathrm{n}}^{*}$ 的变化 $(\phi=1.5)$

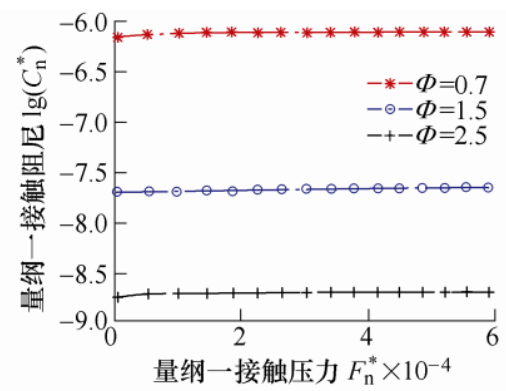

(b) $D=1.26$

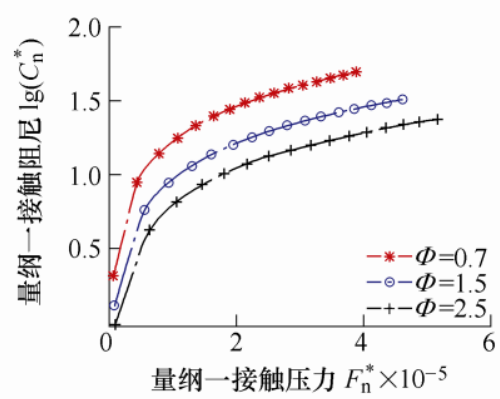

(e) $D=1.56$

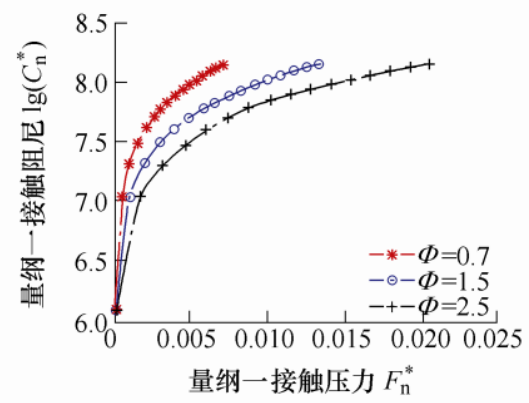

(h) $D=1.86$

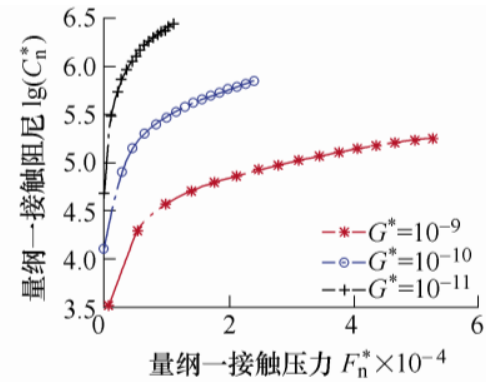

(f) $D=1.66$

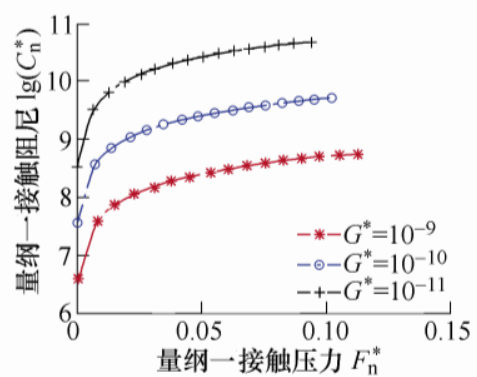

(i) $D=1.96$

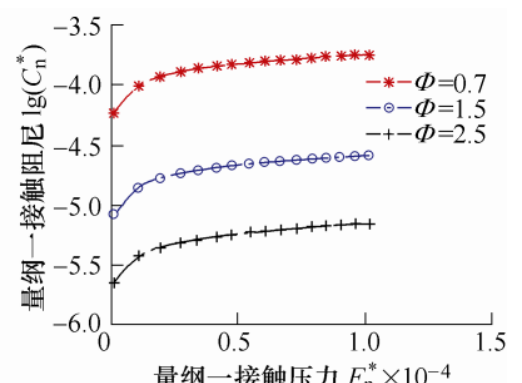

(c) $D=1.36$

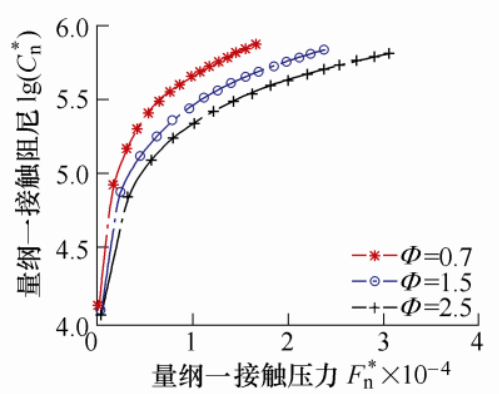

(f) $D=1.66$

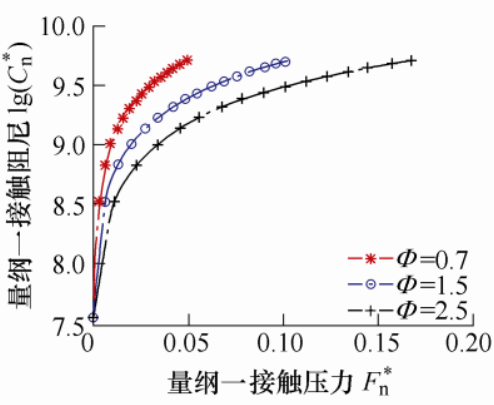

(i) $D=1.96$

图 $5 \lg \left(C_{\mathrm{n}}^{*}\right)$ 随着 $F_{\mathrm{n}}^{*}$ 的变化 $\left(G^{*}=10^{-10}\right)$ 

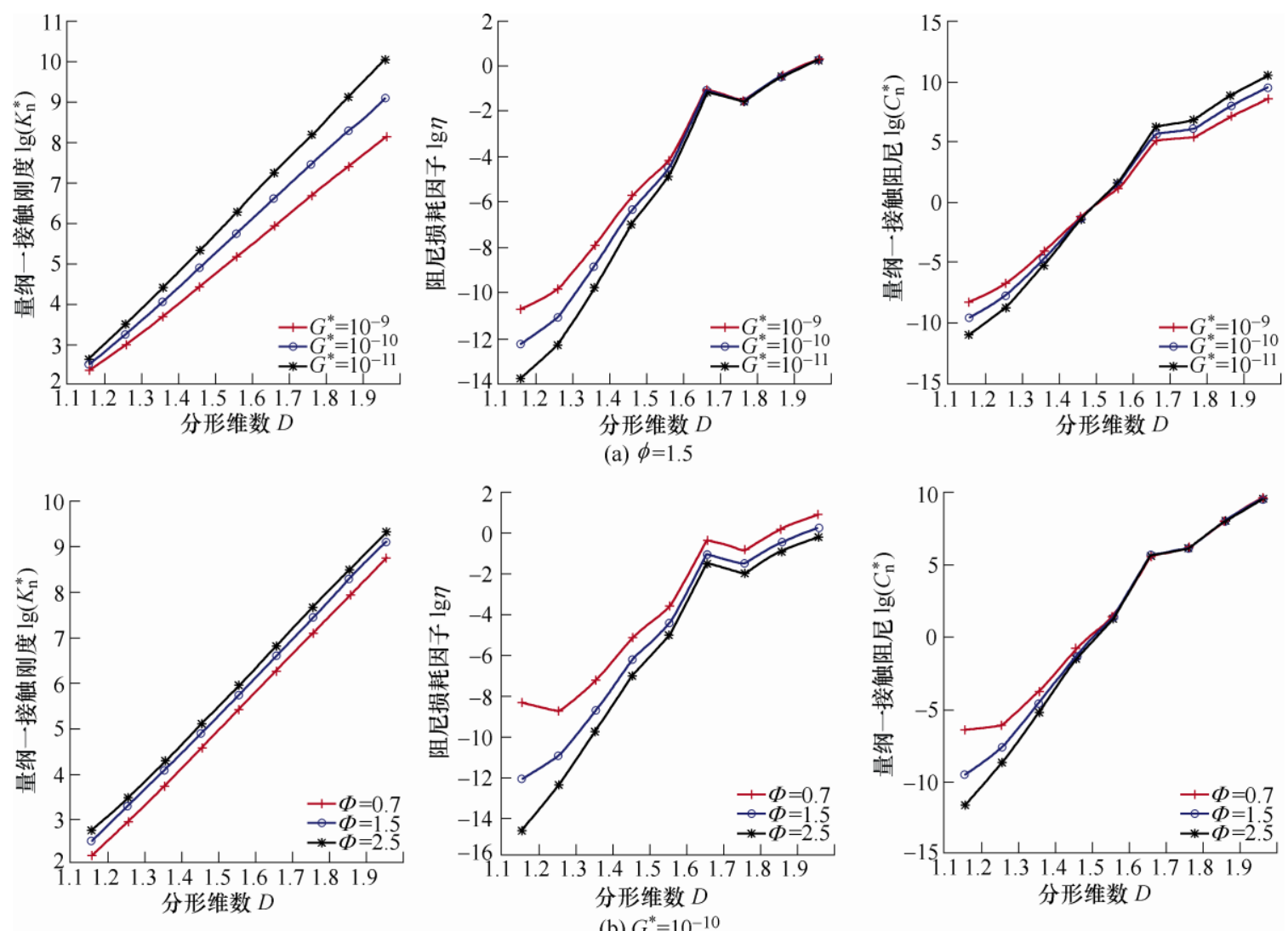

图 $6 D$ 对 $\lg \left(K_{\mathrm{n}}^{*}\right) 、 \lg \eta 、 \lg \left(C_{\mathrm{n}}^{*}\right)$ 的影响

从图 2 中可以看出, 无论 $D$ 为何值, 阻尼损耗 因子的对数 $\lg (\eta)$ 都随量纲一接触载荷 $F_{\mathrm{n}}^{*}$ 的增大而 减小。当 $D \leqslant 1.66$ 时, $\lg \eta$ 随着 $F_{\mathrm{n}}^{*}$ 的增大而发生较 明显的减小, 而当 $D \geqslant 1.66$ 时, 随 $F_{\mathrm{n}}^{*}$ 的增大, $\lg \eta$ 的减小并不太明显, 基本保持不变。另外, 随着 $G^{*}$ 的增大, $\lg \eta$ 呈现出较明显的增大。

从图 3 中可以看出, 当 $D \leqslant 1.66$ 时, $\lg \eta$ 随着 $F_{\mathrm{n}}^{*}$ 的增大而发生较明显的减小, 而当 $D \geqslant 1.66$ 时, 随 $F_{\mathrm{n}}^{*}$ 的增大, $\lg \eta$ 基本保持不变。另外, 随着 $\phi$ 的增 大, $\lg \eta$ 呈现出较明显的减小。

从图 4 中可以看出, 当 $D=1.16$ 时, 量纲一法向 接触阻尼的对数 $\lg \left(C_{\mathrm{n}}^{*}\right)$ 随着 $F_{\mathrm{n}}^{*}$ 的增大而减小; 当 $D=1.26 \sim 1.96$ 时, $\lg \left(C_{\mathrm{n}}^{*}\right)$ 都随 $F_{\mathrm{n}}^{*}$ 的增大而增大。而 且, 当 $D \leqslant 1.36$ 时, $\lg \left(C_{\mathrm{n}}^{*}\right)$ 随着 $G^{*}$ 的增大而明显的 增大, 而当 $D \geqslant 1.46$ 时, $\lg \left(C_{\mathrm{n}}^{*}\right)$ 随着 $G^{*}$ 的增大而明 显的减小。

从图 5 中可以看出, 当 $D=1.16$ 时, $\lg \left(C_{\mathrm{n}}^{*}\right)$ 随 着 $F_{\mathrm{n}}^{*}$ 的增大而减小; 当 $D=1.26 \sim 1.96$ 时, $\lg \left(C_{\mathrm{n}}^{*}\right)$ 都 随 $F_{\mathrm{n}}^{*}$ 的增大而增大, 而且随着 $D$ 的增大, $\lg \left(C_{\mathrm{n}}^{*}\right)$ 的 增大幅度也越大。并且, 无论 $D$ 为何值, $\lg \left(C_{\mathrm{n}}^{*}\right)$ 随 着 $\phi$ 的增大而明显的减小。

从图 6 中可以看出, $D$ 对 $\lg \left(K_{\mathrm{n}}^{*}\right) 、 \lg \eta 、 \lg \left(C_{\mathrm{n}}^{*}\right)$ 的影响, 其中 $D$ 对 $\lg \left(K_{\mathrm{n}}^{*}\right)$ 的影响详见文献[16]。整 体来说, 随着 $D$ 的增大, 无论是 $\lg \eta$ 还是 $\lg \left(C_{\mathrm{n}}^{*}\right)$ 都
是增大的, 但趋势略有不同。图 6a 中可以发现, $D$ 越小, $G^{*}$ 对 $\lg \eta$ 的影响越大, 当 $D \geqslant 1.66$ 时, $G^{*}$ 对 $\lg \left(C_{\mathrm{n}}^{*}\right)$ 的影响很小; 但是, 当 $D \leqslant 1.56$ 时, $G^{*}$ 越大, $\lg \left(C_{\mathrm{n}}^{*}\right)$ 也越大, 当 $D \geqslant 1.56$ 时, $G^{*}$ 越大, $\lg \left(C_{\mathrm{n}}^{*}\right)$ 反 而越小，这与文献[15]中的结论相似，不同之处主 要是由于不同的 $G^{*}$ 所带来的结果。从图 $6 \mathrm{~b}$ 中可以 发现, $D$ 越小, $\phi$ 对 $\lg \eta$ 和 $\lg \left(C_{\mathrm{n}}^{*}\right)$ 的影响越大。当 $D \leqslant 1.66$ 时, $\phi$ 越大, $\lg \left(C_{\mathrm{n}}^{*}\right)$ 就越小; 当 $D \geqslant 1.66$ 时, $\phi$ 对 $\lg \left(C_{\mathrm{n}}^{*}\right)$ 的影响很小。

\section{4 理论计算与试验验证}

在本文试验中，选用浙江景耀数控科技有限公 司的 JNYOLRS250 数控线轨滑台作为试验对象。滚 珠导轨副由四个滑块和两条轨道组成, 一个滑块和 轨道之间的结合部由四列、48 个滚珠(单列 12 个) 与沟槽之间的圆柱面-球面结合面组成, 滚珠与内外 沟槽各有一个圆柱面-球面结合面 ${ }^{[26]}$ 。利用文献[11] 中的结合面参数, 根据式(29)、式(30), 分别计算出 单个滚珠与沟槽之间的结合面法向接触刚度 $K_{\mathrm{n}}$ 和 法向接触阻尼 $C_{\mathrm{n}}$, 在有限元分析时, 选择 combin 14 单元设置滑台与轨道之间的刚度和阻尼参数, 最终 得到滑台在轨道(完全约束)支撑下的理论模态频 率、振型。 
为了验证模型和理论计算结果的正确性, 设计 了线轨滑台的试验模态试验装置, 如图 7 所示。采 用德国 $m+p$ 5800B4 模态力锤激励, 12 个德国 $m+p$ 3035BG 单轴压电式加速度传感器, 德国 $m+p$ VibRunner32Ch 数据采集仪及试验模态分析系统, 得到滑台的试验模态频率、模态阻尼和振型(仅包含 垂直方向)。

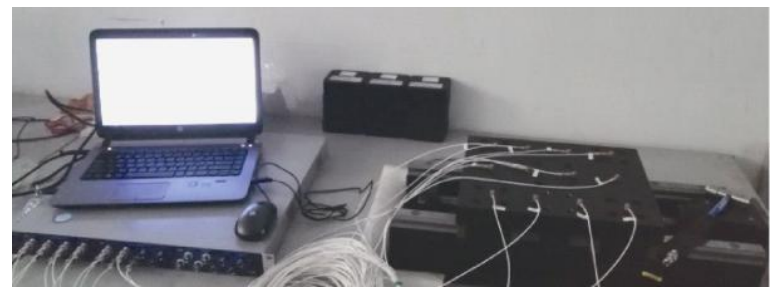

图 7 线轨滑台试验装置

以模态振型(部分振型见图 8)相同为比较原则, 将理论模态和试验模态识别的固有频率进行了比

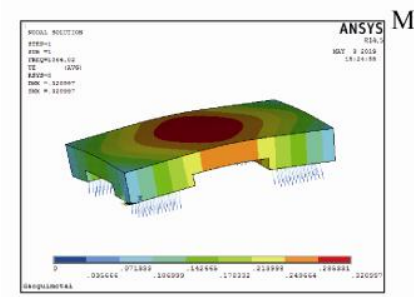

(a) $f_{1}=1064.202 \mathrm{~Hz}$

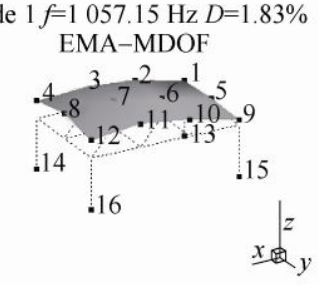

(b) $f_{1}=1057.15 \mathrm{~Hz}$

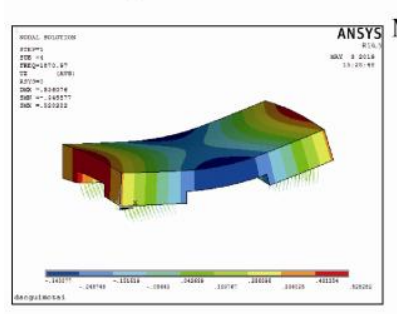

(c) $f_{2}=1870.97 \mathrm{~Hz}$

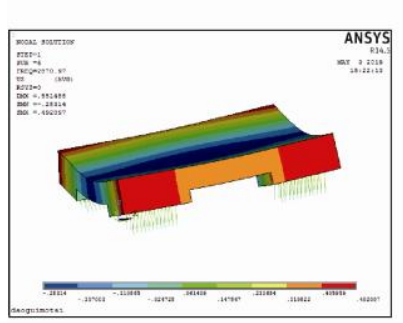

(e) $f_{3}=2870.97 \mathrm{~Hz}$

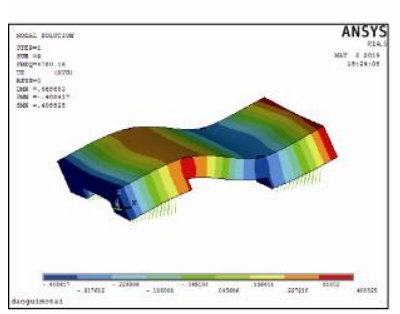

(g) $f_{4}=4780.16 \mathrm{~Hz}$

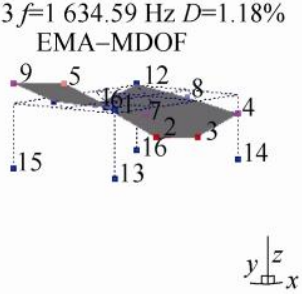

(d) $f_{2}=1634.59 \mathrm{~Hz}$

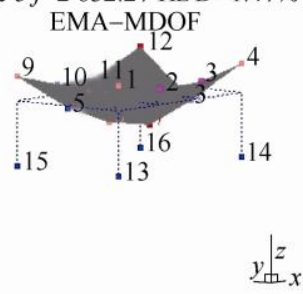

(f) $f_{3}=2632.27 \mathrm{~Hz}$ Mode $7 f=4328.66 \mathrm{~Hz} D=1.46 \%$

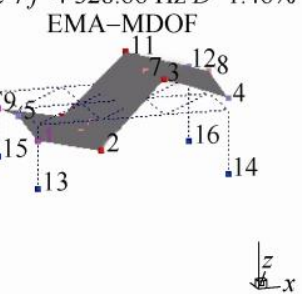

(h) $f_{4}=4328.66 \mathrm{~Hz}$ Mode $5 f=2632.27 \mathrm{~Hz} D=1.47 \%$

图 8 理论计算与试验模态振型比较
较, 见表 1 。在振型相同时, 理论计算与试验模态 参数识之间存在误差, 误差最小为 $0.65 \%$ (固有频率 为 $1057.15 \mathrm{~Hz}$ ), 最大为 $14.43 \%$ (固有频率为 1634.59 $\mathrm{Hz}$ ), 从图 9 中也可以看出, 固有频率为 $1634.4 \mathrm{~Hz}$ 时的相干系数为 0.814(其余均超过 0.94), 相对较低, 说明在试验模态参数识别时有一定的误差, 这也是 造成误差较大的原因之一。对于机械结构而言，低 阶固有频率比高阶固有频率更重要，对计算精度的 要求也更高, 因此, 利用本文提出的法向接触刚度 模型与法向接触阻尼模型来计算结构的低阶模态频 率, 结果较为准确。

表 1 理论计算与试验模态识别固有频率比较

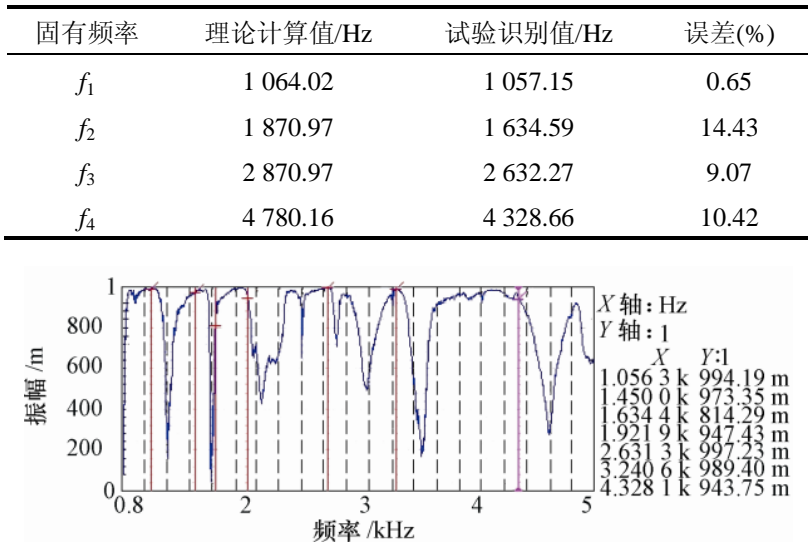

图 9 响应点 9 与激励点 7 之间的相干函数

\section{5 结论}

（1）在文献[16]的基础上, 分析了微凸体接触的 弹塑性阶段的特点, 并将法向接触载荷 “分离” 为 弹性载荷和塑性载荷, 从而得到微凸体接触在三个 阶段的弹性能和损耗能。并将结合面法向接触动力 学模型等效为弹簧和黏性阻尼器, 建立了包含弹塑 性阶段的结合面法向阻尼损耗因子模型和法向接触 阻尼模型, 并进行了量纲一化处理, 得到了结合面 阻尼损耗因子和量纲一法向接触阻尼模型。本文建 立的结合面法向接触阻尼模型是解析的, 可以为实 际设计提供理论支持。

(2) 通过仿真发现, 在分形维数较小时, 分形 粗粘度参数和塑性指数对结合面阻尼损耗因子和法 向接触阻尼的影响较大; 而当分形维数较大时, 分 形粗鋉度参数和塑性指数对阻尼损耗因子和法向接 触阻尼的影响都较小。

(3) 分形维数是影响阻尼损耗因子和法向接触 阻尼的最主要因数, 在塑性指数和分形粗糙度一定 时, 阻尼损耗因子和法向接触阻尼变化是最大的。 在塑性指数一定的情况下，随着分形维数的增大， 
分形粗糙度对法向接触阻尼的影响与分形维数的大 小有关: 当表面粗粘轮廓分形维数小于拐点值 ( $D=1.56$ 左右) 时, 法向接触阻尼随着分形粗粘度的 增大而增大; 当分形维数超过拐点值时, 呈现出分 形粗粘度越大，法向接触阻尼反而减小的现象。以 上结合面法向接触阻尼的变化趋势和特点与文献 [15]中图 4a 以及文献[27]中图 8 的结论近似相同。 与之相应是, 当分形粗粘度一定时, 随着分形维数 的增大, 塑性指数对法向接触阻尼的影响只发生在 分形维数较小时 $(D<1.66)$, 塑性指数越大, 法向接 触阻尼反而越小; 当 $D>1.66$ 时, 塑性指数的变化 基本不影响法向接触阻尼。

(4) 本文结合面法向接触参数模型计算的理论 模态固有频率与试验模态识别的固有频率进行比 较, 发现低阶固有频率的准确性较高, 对于机械结 构结合面动力学分析有一定的指导意义。

\section{参 考 文 献}

[1] 张学良. 机械结合面动态特性及应用 [M]. 北京: 中国 科技出版社，2002.

ZHANG Xueliang. Dynamic characteristics of machine joint surfaces and its applications[M]. Beijing: Press of Science and Technology of China, 2002.

[2] 张广鹏, 史文浩, 黄玉美, 等. 机床整机动态特性的预 测解析建模方法 [J]. 上海交通大学学报, 2001, 35(12): 1834-1837.

ZHANG Guangpeng, SHI Wenhao, HUANG Yumei, et al. Modeling and analysis method of dynamical characteristics for a whole machine tool structure[J]. Journal of Shanghai Jiaotong University，2001，35(12): 1834-1837.

[3] 张广鹏, 史文浩, 黄玉美. 机床导轨结合部的动态特性 解析方法及其应用 [J]. 机械工程学报, 2002, 38(10): 114-117.

ZHANG Guangpeng, SHI Wenhao, HUANG Yumei. Analysis method of dynamic behaviors of guidway joint and its application in machine tools design[J]. Chinese Journal of Mechanical Engineering, 2002, 38(10): 114-117.

[4] ZHAO Yongwu, DAVID D M, CHANG L. An asperity microcontact model incorporating the transition from elastic deformation to fully plastic flow[J]. ASME Journal of Tribology, 2000, 122(1): 86-93.

[5] KOGUT L, ETSION I. Elastic-plastic contact analysis of a sphere and rigid flat[J]. Journal of Applied Mechanics, 2002, 69(5): 657-662.

[6] KOGUT L , ETSION I. A finite element based elastic-plastic model for the contact of rough surfaces[J].
Tribology Transactions, 2003, 46(3): 383-390.

[7] 赵永武, 吕彦明, 蒋建忠. 新的粗糙表面弹塑性接触模 型[J]. 机械工程学报, 2007, 43(3): 95-101.

ZHAO Yongwu, LÜ Yanming, JIANG Jianzhong. New elastic-plastic model for the contact of rough surface[J]. Chinese Journal of Mechanical Engineering, 2007, 43(3): 95-101.

[8] BRAKE M. An analytical elastic-perfectly plastic contact model[J]. International Journal of Solids and Structures, 2012, 49(22): 3129-3141.

[9] 徐超, 王东. 一种改进的粗䊁表面法向弹塑性接触解析 模型[J]. 西安交通大学学报, 2014, 48(44): 115-121.

XU Chao, WANG Dong. An improved analytical model for normal elastic-plastic contact of rough surfaces[J]. Journal of Xi'an Jiaotong University, 2014, 48(44): 115-121.

[10] JIANG Shuyun, ZHENG Yunjian, ZHU Hua. A contact stiffness model of machined plane joint based on fractal theory[J]. Journal of Tribology, 2010, 132(1): 011401 -011407 .

[11] 张学良, 陈永会, 温淑花, 等. 考虑弹塑性变形机制的 结合面法向接触刚度建模 [J]. 振动工程学报, 2015, 28(1): 91-99.

ZHANG Xueliang, CHEN Yonghui, WEN Shuhua, et al. The model of normal contact stiffness of joint interfaces incorporating elastoplastic deformation mechanism[J]. Journal of Vibration Engineering，2015，28(1): 91-99.

[12] RAFFA M L, LEBON F, VAIRO G. Normal and tangential stiffnesses of rough surfaces in contact via an imperfect interface model[J]. International Journal of Solids and Structures, 2016, 87(6): 245-253.

[13] 王南山, 张学良, 兰国生, 等. 临界接触参数连续的粗 糙表面法向接触刚度弹塑性分形模型 $[\mathrm{J}]$. 振动与冲击, 2014, 33(9): 72-77.

WANG Nanshan, ZHANG Xueliang, LAN Guosheng, et al. Elastoplastic fractal model for normal contact stiffness of rough surfaces with continuous contact parameters[J]. Journal of Vibration and Shock, 2014, 33(9): 72-77.

[14] 尤晋闽, 陈天宁. 结合面法向动态参数的分形模型 $[\mathrm{J}]$. 西安交通大学学报，2009，43(9): 91-94.

YOU Jinmin, CHEN Tianning. Fractal model for normal dynamic parameters of joint surfaces[J]. Journal of Xi'an Jiaotong University, 2009, 43(9): 91-94.

[15] 田红亮，赵美云，郑金华，等. 新的柔性结合部法向接 触刚度和接触阻尼方程 $[\mathrm{J}]$. 西安交通大学学报, 2015, 49(1): 118-126.

TIAN Hongliang, ZHAO Meiyun, ZHENG Jinhua, et al. New equations of normal contact stiffness and damping 
for flexible joint interface[J]. Journal of Xi'an Jiaotong University, 2015, 49(1): 118-126.

[16] 陈永会, 张学良, 温淑花, 等. 粗糙表面弹塑性接触连 续光滑指数函数模型与法向接触刚度研究 $[\mathrm{J}]$. 西安交 通大学学报, 2016, 50(7): 58-67.

CHEN Yonghui, ZHANG Xueliang, WEN Shuhua, et al. Research on continuous smooth exponential model of elastic-plastic contact and normal contact stiffness of rough surface $[\mathrm{J}]$. Journal of Xi'an Jiaotong University, 2016, 50(7): 58-67.

[17] GOODMAN L E, BOWIE G E. Experiments on damping at contacts of a sphere with flat plates[J]. Experimental Mechanics, 1961, 1(2): 48-54.

[18] SHI Xi , POLYCARPOU A A. Measurement and modeling of normal contact stiffness and contact damping at the meso scale[J]. Journal of Vibration and Acoustics, 2005, 127(2): 52-60.

[19] SHI Xi, POLYCARPOU A A. Investigation of contact stiffnessand contact damping for magnetic storage Head-Disk interfaces[J]. Journal of Tribology, 2008, 130(2): 843-854.

[20] 张学良, 丁红钦, 兰国生, 等. 基于分形理论的结合面 法向接触阻尼与损耗因子模型 $[\mathrm{J}]$. 农业机械学报, 2013, 44(6): 287-294.

ZHANG Xueliang, DING Hongqin, LAN Guosheng, et al. Normal contact damping and dissipation factor model of joint interfaces based on fractal theory[J]. Transactions of the Chinese Society for Agricultural Machinery, 2013, 44(6): 287-294.

[21] 田红亮, 郑金华, 赵春华, 等. 界面损耗因子与法向阻 尼的计算方法 $[J]$. 上海交通大学学报, 2015, 49(5): 687-694.

TIAN Hongliang, ZHENG Jinhua, ZHAO Chunhua, et al. Calculating method of surface dissipation factor and normal damping[J]. Journal of Shanghai Jiaotong University, 2015, 49(5): 687-694.

[22] GREENWOOD J A, TRIPP J H. The contact of two nominally flat rough surfaces $[\mathrm{J}]$. Proceedings of the Institution of Mechanical Engineers, 1970, 185(1): 625-633.

[23] MJUMDAR A , BHUSHAN B. Fractal model of elastic-plastic contact between rough surfaces [J]. Journal of Tribology, 1991, 113 (1): 1-11.

[24] 傅卫平, 娄雷亭, 高志强, 等. 机械结合面法向接触刚 度和阻尼的理论模型 $[J]$. 机械工程学报, 2017, 53(9): 73-82.

FU Weiping, LOU Leiting, GAO Zhiqiang, et al. Theoretical model for the contact stiffness and damping of mechanical joint surface[J]. Journal of Mechanical Engineering, 2017, 53(9): 73-82.

[25] KONOWALSKI K. Experimental research and modeling of normal contact stiffness and contact damping of machined joint surfaces[J]. Advances in Manufacturing Science and Technology, 2009, 33(3): 53-68.

[26] 刘耀, 黄玉美. 机床滚珠导轨中圆柱面-球面结合面静 特性分析及试验研究[J]. 机械工程学报, 2013, 49(21): 25-30.

LIU Yao, HUANG Yumei. Theoretical analysis and experimental study on static characteristics of the cylindrical-spherical Joint Surfaces of linear ball guide on machine tool[J]. Journal of Mechanical Engineering, 2013, 49(21): 25-30.

[27] LIU Wenwei, YANG Jiajun, XI Neng, et al. A study of normal dynamic parameter models of joint interfaces based on fractal theory[J]. Journal of Advanced Mechanical Design Systems\& Manufacturing, 2015, 9(5): JAMDSM0070 1-13.

作者简介: 陈永会, 男, 1975 年出生, 博士研究生, 副教授。主要研究 方向为结合面接触特性、机械结构动力学。

E-mail: tycyh9672@163.com

张学良(通信作者), 男, 1964 年出生, 博士, 教授, 博士研究生导师。 主要研究方向为机械结合面接触特性、智能优化算法及应用、机电系统 结构动态特性与测试技术。

E-mail: zhang_xue_1@sina.com 